\title{
Coexistance of giant tunneling electroresistance and magnetoresistance in an all-oxide magnetic tunnel junction
}

\author{
Nuala Mai Caffrey, Thomas Archer, Ivan Runnger, and Stefano Sanvito \\ School of Physics and CRANN, Trinity College, Dublin 2, Ireland
}

(Dated: September 14, 2018)

\begin{abstract}
We demonstrate with first-principles electron transport calculations that large tunneling magnetoresistance (TMR) and tunneling electroresistance (TER) effects can coexist in an all-oxide device. The TMR originates from the symmetry-driven spin filtering provided by the insulating $\mathrm{BaTiO}_{3}$ barrier to the electrons injected from $\mathrm{SrRuO}_{3}$. In contrast the TER is possible only when a thin $\mathrm{SrTiO}_{3}$ layer is intercalated at one of the $\mathrm{SrRuO}_{3} / \mathrm{BaTiO}_{3}$ interfaces. As the complex band-structure of $\mathrm{SrTiO}_{3}$ has the same symmetry than that of $\mathrm{BaTiO}_{3}$, the inclusion of such an intercalated layer does not negatively alter the TMR and in fact increases it. Crucially, the magnitude of the TER also scales with the thickness of the $\mathrm{SrTiO}_{3}$ layer. The $\mathrm{SrTiO}_{3}$ thickness becomes then a single control parameter for both the TMR and the TER effect. This protocol offers a practical way to the fabrication of four-state memory cells.
\end{abstract}

Epitaxial magnetic tunnel junctions (MTJs), displaying giant tunneling magnetoresistance (TMR) at room temperature [1, 2], represent the enabling technology for ultra-high density magnetic data storage. In MTJs the insulating barrier plays a dual role; it magnetically decouples the two ferromagnetic (FM) electrodes so that their magnetizations, $M$, can be arranged either parallel or antiparallel to each other, but it can also act as a spin filter, if epitaxially grown. This is due to the wavefunction symmetry selective decay of tunneling electrons across a crystalline insulator. As the two spin-manifolds of the Fermi surface of a FM metal present different symmetries, such symmetry selectivity translates into spin selectivity. This is the case for $\mathrm{Fe} / \mathrm{MgO}(001)$, where the barrier is more transparent to the tunneling of electrons with $\Delta_{1}$ symmetry. These are present in Fe only for the majority spin [3, 4], so that the $\mathrm{Fe} / \mathrm{MgO}(001)$ stack effectively behaves as a half-metal.

In conventional MTJs, however, the insulating barrier is a passive element, i.e., its electronic structure cannot be changed by external stimuli. A different situation is encountered when using a ferroelectric (FE) material. A FE is intrinsically insulating and at the same time possesses a macroscopic order, the electrical polarization, $P$. When FE materials are incorporated into a tunnel junction, one expects the junction resistance to become dependent on the direction of $P$ with respect to the layer stack, an effect known as tunneling electroresistance (TER) [5]. It then becomes natural to think about devices combining materials with both FM and FE orderings [6]. Here, one can exploit the possibility of manipulating the two independent order parameters, $P$ and $M$, by means of their conjugate fields, namely the electric and magnetic fields. The fabrication of FE random access memories with non-destructive reading [7] demonstrates the potential of such an approach.

Although it is possible, at least in principle, to obtain a large TMR in MTJs with FE barriers [8, 9], it is sensibly more complicated to obtain a large TER. The key ingredient for a MTJ to show TER is that it should exhibit inversion symmetry breaking. This is almost always the case in real devices as unintentional disorder breaks the symmetry. However, disorder is hardly controllable. Furthermore, even when the entire junction termination is different at either side of the insulating barrier the TER appears relatively modest [9]. A second strategy uses two magnetic electrodes made of different metals and thus different abilities to screen surface charges [5]. In typical metals with high carrier mobility, however, the screening length is short and the surface charge is strongly localized at the interface. The resulting potential profile thus remains approximately mirrorsymmetric upon polarization reversal and the expected TER is small. Even for $\mathrm{Fe} / \mathrm{BaTiO}_{3} / \mathrm{La}_{0.67} \mathrm{Sr}_{0.33} \mathrm{MnO}_{3}$ junctions, where $\mathrm{La}_{0.67} \mathrm{Sr}_{0.33} \mathrm{MnO}_{3}$ is quite a poor metal, a TER of only $37 \%$ has been observed [10].

One can bring the concept of having different screening lengths at the two sides of the FE layer to the extreme by including a second insulator (INS) in the stack, i.e., by considering a FM/INS/FE/FM junction. An interesting example of such structure is when the INS is actually vacuum, as in a scanning tunnel microscopy experiment. Here, an extremely large TER has already been measured 11, 12. In this letter we demonstrate, by first principles electronic transport calculations, that the same effect can be achieved in an all-oxide solid state device. In particular, we show that intercalating a few monolayers of $\mathrm{SrTiO}_{3}$ into a $\mathrm{SrRuO}_{3} / \mathrm{BaTiO}_{3} / \mathrm{SrRuO}_{3}$ junction creates an additional potential barrier that is switchable with the FE polarization of $\mathrm{BaTiO}_{3}$. This not only amplifies the TER, but also makes it exponentially dependent on the $\mathrm{SrTiO}_{3}$ thickness. Furthermore, as $\mathrm{SrTiO}_{3}$ is electronically very similar to $\mathrm{BaTiO}_{3}$ and thereby provides comparable spin-filtering for $\mathrm{SrRuO}_{3}$ [8, 9], the junction also displays a remarkably large TMR.

The electronic structure of the junction is calculated by using density functional theory (DFT) as numerically implemented in the SIESTA code [13]. Structural relax- 
ation is performed with the Perdew-Burke-Ernzerhof generalized gradient approximation (GGA) 14 to the exchange and correlation functional, with a $6 \times 6 \times 1 k$-point Monkhorst-Pack mesh and a grid spacing equivalent to a plane-wave cutoff of $800 \mathrm{eV}$. In contrast, for the electronic properties and the transport we use the atomic self-interaction correction (ASIC) scheme [15] built over the local spin density approximation. ASIC has been previously found to improve the electronic properties of bulk $\mathrm{BaTiO}_{3}$ [16] and $\mathrm{SrRuO}_{3}$ [17] and it is vital in transport calculations where one has to ensure a good band alignment between dissimilar materials [18]. Unfortunately, even in its variational form [19] the ASIC scheme does not describe the FE phase accurately enough, so that a compromise is required; the GGA is used for the relaxation and the ASIC for the transport calculations. Electron transport is computed with the $a b$ initio code SMEAGOL [20 22], which combines DFT with the non-equilibrium Green's functions scheme. SMEAGOL uses SIESTA as its DFT platform so that the same convergence parameters are employed for the transport, except for the $k$-point sampling where we consider a much larger $100 \times 100 \times 1$ mesh.

The supercell considered here comprises $6 \mathrm{BaTiO}_{3}$ unit cells $(\sim 2.5 \mathrm{~nm})$ and 3 unit cells of $\mathrm{SrRuO}_{3}$ attached at each side to function as electrodes. Furthermore, we intercalate a thin $\mathrm{SrTiO}_{3}$ layer between $\mathrm{BaTiO}_{3}$ and $\mathrm{SrRuO}_{3}$ at one side of the junction so that that the final stack is $\left(\mathrm{SrO}-\mathrm{RuO}_{2}\right)_{3} /\left(\mathrm{SrO}-\mathrm{TiO}_{2}\right)_{m} /(\mathrm{BaO}-$ $\left.\mathrm{TiO}_{2}\right)_{6} /\left(\mathrm{SrO}-\mathrm{RuO}_{2}\right)_{3}$, where $m=0,1,2$. The in-plane lattice parameter is set to that of bulk $\mathrm{SrTiO}_{3}(3.95 \AA)$ to mimic the effect of a $\mathrm{SrTiO}_{3}$ substrate. This applies compressive strain to both $\mathrm{SrRuO}_{3}$ and $\mathrm{BaTiO}_{3}$ and in doing so increases the polarization of $\mathrm{BaTiO}_{3}$. The Berry phase method gives a GGA polarization of $43.8 \mu \mathrm{C} / \mathrm{cm}^{2}$ for the bulk $(c / a=1.05)$ and $48.1 \mu \mathrm{C} / \mathrm{cm}^{2}$ for the strained structure $(c / a=1.08)$. Note that the GGA systematically overestimates the polarization of FE oxides, but such a detail does not affect our conclusions.

We consider two alternative geometries for the junction, characterized by the $\mathrm{BaTiO}_{3}$ polarization pointing in opposite directions. Both geometries are relaxed to a tolerance of $40 \mathrm{meV} / \AA$ (less than $4 \mathrm{meV} / \AA$ for the $m=0$ case). When $\mathrm{BaTiO}_{3}$ is included in the capacitor structure the displacements at the center of the supercell correspond to a polarization of $35.5 \mu \mathrm{C} / \mathrm{cm}^{2}$, i.e., sensibly reduced from its bulk value.

An indication of the polarization structure is obtained from Fig. 1] where we show the atomic displacements, $\delta$, along the MTJ stack. Here $\delta=\left(z_{\text {cation }}-z_{\mathrm{O}}\right)$, where $z_{\text {cation }}$ and $z_{\mathrm{O}}$ denote respectively the cation and $\mathrm{O}$ position in a particular plane. As such, $\delta>0$ defines a structure with the polarization pointing parallel to the substrate normal and away from the intercalated $\mathrm{SrTiO}_{3}$ layer $\left(P_{\rightarrow}\right)$, while $\delta<0$ define a structure with the polarization pointing in the opposite direction $\left(P_{\leftarrow}\right)$. Clearly,
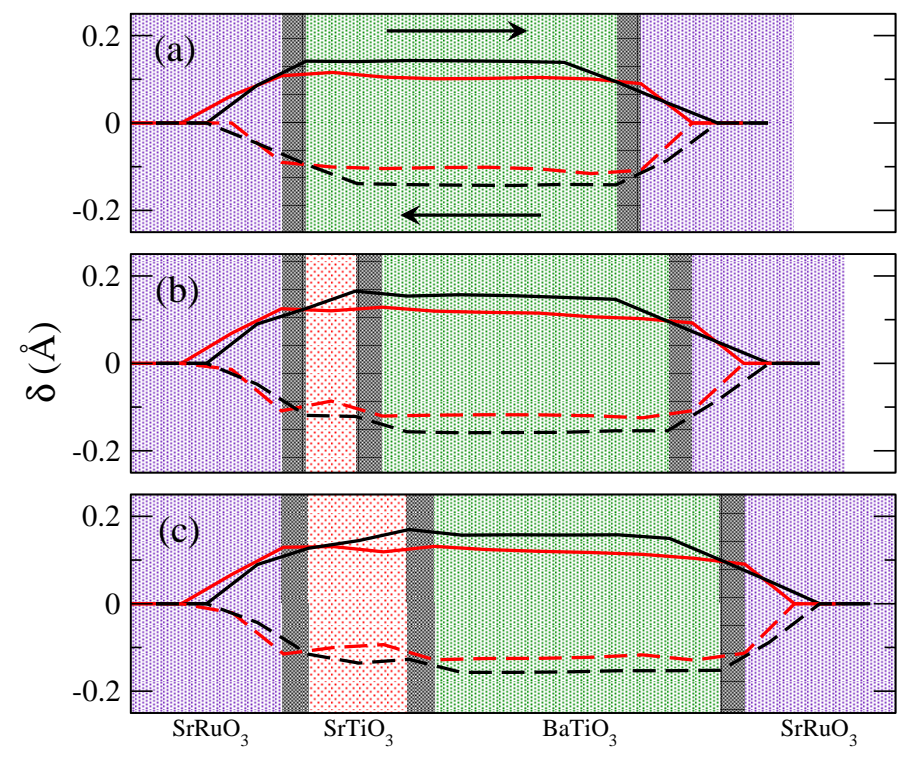

FIG. 1. Relative cation-oxygen displacements along the $z$-axis (the junction stack direction) for the fully relaxed structure: (a) $m=0$, (b) $m=1$ and (c) $m=2$. The black (red) line corresponds to displacements in the $\mathrm{BO}_{2}(\mathrm{AO})$ planes, of $\mathrm{ABO}_{3}$. The solid (dashed) lines indicate $P_{\rightarrow}\left(P_{\leftarrow}\right)$.

as far as the displacement is concerned there are no significant differences between the intercalated $\mathrm{SrTiO}_{3}$ and $\mathrm{BaTiO}_{3}$, which means that $\mathrm{SrTiO}_{3}$, an incipient FE, takes on the $\mathrm{FE}$ distortion of $\mathrm{BaTiO}_{3}$. This is valid only for thin $\mathrm{SrTiO}_{3}$ films $(m=1,2)$ while we expect that thicker layers will lose the FE state.
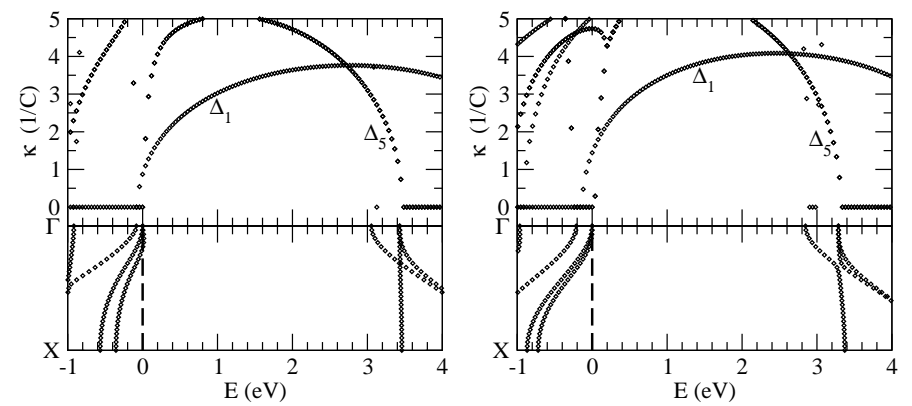

FIG. 2. Complex and real band structure of bulk $\mathrm{SrTiO}_{3}$ (left panel) and bulk $\mathrm{BaTiO}_{3}$ (right panel), calculated for the FE structure constrained to the in-plane lattice parameter of $\mathrm{SrTiO}_{3}$.

The electronic structures of $\mathrm{SrTiO}_{3}$ and $\mathrm{BaTiO}_{3}$ are also rather similar to each other as shown in Fig. 2, where one can observe that the real band structures of the two materials almost coincide. Furthermore, and more importantly here, the symmetry of the complex part of the band structure is identical in both materials, with a $\Delta_{1}$ symmetry band dominating the lower energy part of the band gap and a $\Delta_{5}$ one defining the region near to the conduction band. We then expect that interca- 
lating a $\mathrm{SrTiO}_{3}$ layer will give a MTJ with the same spin-filtering properties of the $\mathrm{SrRuO}_{3} / \mathrm{BaTiO}_{3} / \mathrm{SrRuO}_{3}$ stack [8]. Here, we use the "optimistic" TMR ratio, $R_{\mathrm{TMR}}=\frac{G_{\uparrow \uparrow}-G_{\uparrow \downarrow}}{G_{\uparrow \downarrow}}$, where $G_{\uparrow \uparrow}\left(G_{\uparrow \downarrow}\right)$ is the total conductance for the parallel (antiparallel) orientation of the magnetization. The TMR is found to increase with $\mathrm{SrTiO}_{3}$ thickness due to the increasing length of the spinfiltering barrier. In particular, for $m=2$ at zero-bias $R_{\text {TMR }}$ exceeds $10^{8 \%}$ (the actual value depending on the polarization direction), meaning that at these thicknesses the barrier acts as an almost perfect spin-filter.

We now discuss the TER effect in the junction by first looking at the electrostatic potential profile. In order to sustain the internal electric field associated with a FE material, the electrostatic potential profile must display a finite slope. Concurrently, assuming that no bias is applied across the junction and that the two electrodes are made of identical materials, i.e., they have the same Fermi energy, the average potential in the electrodes should be identical. As a consequence, it is necessary that surface charge forms at the interface between the FE layer and the metallic electrodes. This creates a depolarizing field so that the potential across the interface can be matched and also sets the critical thickness for the onset of the FE state in a thin film [23].
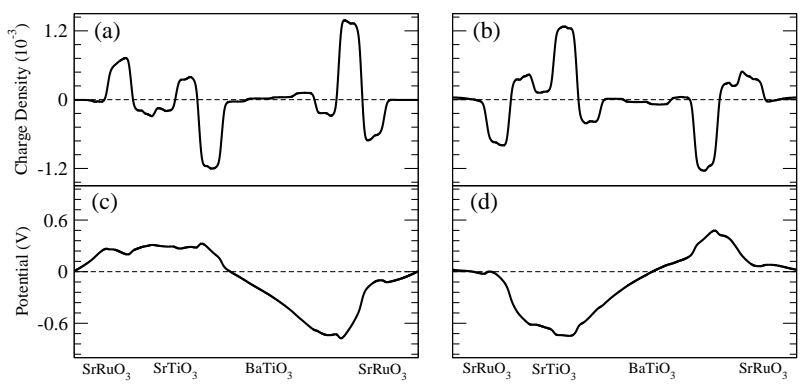

FIG. 3. Difference in charge distribution and electrostatic potential profile through the $m=2$ junction. These are the differences between the relative quantities (charge density and electrostatic potential) as calculated for the FE and centrosymmetric structures and averaged over the plane perpendicular to the junction stack. The left (right) panel is for $P \rightarrow\left(P_{\leftarrow}\right)$.

In Fig. 3 we present both the charge density and the electrostatic potential profile across the $m=2$ junction. These are obtained as the planar average of the difference between the relevant quantity calculated for the centrosymmetric and FE configurations. The atomic oscillations thereby cancel and one is left with the modifications of the charge density and the potential due to the onset of the FE phase. In general we observe that charge density of opposite sign forms at either side of the FE layer resulting in the expected potential difference. As we move into the metallic layers at the $\mathrm{BaTiO}_{3} / \mathrm{SrRuO}_{3}$ interface an additional peak in the charge density can be seen, which acts as a depolarization charge and brings the potential back to zero. In contrast, at the $\mathrm{SrTiO}_{3} / \mathrm{BaTiO}_{3}$ interface there are not sufficient screening charges so that the depolarization charge forms instead at the metallic $\mathrm{SrRuO}_{3}$ electrode. This leaves the potential in $\mathrm{SrTiO}_{3}$ pinned to that at the interface with $\mathrm{BaTiO}_{3}$. Thus, when one reverses $P$ the potential in the $\mathrm{SrTiO}_{3}$ layer is rigidly shifted. Note that, as a consequence of such charge distribution, the average electrostatic potential in $\mathrm{SrTiO}_{3}$ remains flat despite the ionic displacement.

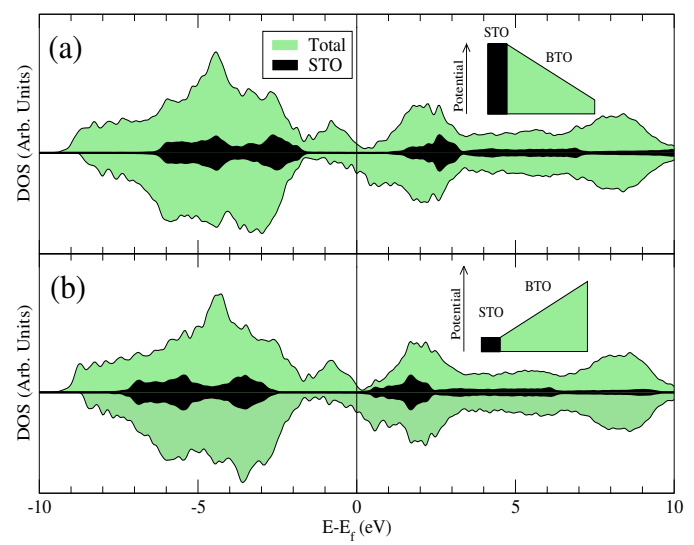

FIG. 4. Total density of states (DOS) for the $m=2$ junction (green shaded area) and DOS projected over the $\mathrm{SrTiO}_{3}$ layer (solid black area) for the two different polarization directions, namely (a) $P_{\rightarrow}$ and (b) $P_{\leftarrow}$. Note the rigid shift in the $\mathrm{SrTiO}_{3}$ DOS as $P$ is reversed. In the insets we show a schematic of the barrier profile for both polarization directions.

This rigid shift in the potential can be appreciated by looking at Fig. 4, where we show that the density of states (DOS) projected onto the $\mathrm{SrTiO}_{3}$ layer is rigidly displaced by the reversal of the $\mathrm{BaTiO}_{3}$ polarization direction. In particular, for the $P_{\leftarrow}$ configuration the $\mathrm{SrTiO}_{3}$ conduction band edge is considerably closer to the junction Fermi level, $E_{\mathrm{F}}$, than for the $P_{\rightarrow}$ case. This means that the height of the $\mathrm{SrTiO}_{3}$ potential barrier presented to the tunneling electrons changes according to the direction of $P$. In summary, the overall scattering potential appears as follows: for $P_{\rightarrow}$ there is a high barrier in $\mathrm{SrTiO}_{3}$ followed by a triangular barrier in $\mathrm{BaTiO}_{3}$, which decreases as one moves away from the $\mathrm{TiO}_{2} / \mathrm{BaO}$ interface [see insets of Fig. 4(a)]. In contrast, for $P_{\leftarrow}$ the $\mathrm{SrTiO}_{3}$ barrier is small while the triangular $\mathrm{BaTiO}_{3}$ barrier increases away from the interface. As a consequence, tunneling through $\mathrm{BaTiO}_{3}$ is essentially insensitive to the polarization direction, as the two triangular barriers are identical. However, the barrier height across $\mathrm{SrTiO}_{3}$ changes significantly with the $P$ direction. Such a polarization-dependent change in the $\mathrm{SrTiO}_{3}$ barrier is the cause of the TER effect in this junction.

In Table@ we summarize our transport results. In particular, we present the junction conductance at zero bias 


\begin{tabular}{ccccccc}
\hline \hline$m$ & $\sigma \sigma^{\prime}$ & $G_{\rightarrow}^{\sigma \sigma^{\prime}}$ & $G_{\leftarrow}^{\sigma \sigma^{\prime}}$ & $R_{\mathrm{TER}}^{\sigma \sigma^{\prime}}$ & $R_{\mathrm{TMR}}^{\rightarrow}$ & $R_{\mathrm{TMR}}^{\leftarrow}$ \\
\hline 0 & $\uparrow \uparrow$ & $4.05 \times 10^{6}$ & $4.06 \times 10^{6}$ & 0.31 & & \\
\hline 1 & $\uparrow \uparrow$ & $6.82 \times 10^{4}$ & $9.49 \times 10^{4}$ & 39.07 & & \\
\hline 2 & $\uparrow \uparrow$ & $2.86 \times 10^{3}$ & $8.95 \times 10^{3}$ & 212.84 & & \\
2 & $\uparrow \downarrow$ & $1.12 \times 10^{-4}$ & $1.83 \times 10^{-3}$ & 1533.93 & $2.5 \times 10^{9}$ & $4.75 \times 10^{8}$ \\
\hline \hline
\end{tabular}

TABLE I. Layer conductance (in units of $\Omega^{-1} \mathrm{~cm}^{-2}$ ) and both TMR and TER ratios (in \%) for different $m$. Here $G_{\alpha}^{\sigma \sigma^{\prime}}$ is the layer conductance for the magnetic configuration $\sigma \sigma^{\prime}$ and the electrical polarization pointing in the $\alpha$ direction. Note that the TER depends on the magnetic configuration of the junction and the TMR depends on the electrical configuration.

for the two different $P$ directions (either $\leftarrow$ or $\rightarrow$ ) and the two different magnetic arrangements of the electrodes (either parallel $\uparrow \uparrow$ or antiparallel $\uparrow \downarrow$ orientation) for $m=0,1$ and 2 . In the table, in addition to $R_{\mathrm{TMR}}$, we also report the figure of merit for the TER effect, namely the TER ratio $R_{\mathrm{TER}}^{\sigma \sigma^{\prime}}=\frac{G_{\leftarrow}^{\sigma \sigma^{\prime}}-G^{\sigma \sigma^{\prime}}}{G^{\sigma \sigma^{\prime}}}$. Note that the TMR is now dependent on the polarization direction so the ratio is defined as $R_{\mathrm{TMR}}^{\alpha}=\frac{G_{\alpha}^{\uparrow \uparrow}-G_{\alpha}^{\uparrow \downarrow}}{G_{\alpha}^{\uparrow \downarrow}}$, being $G_{\alpha}^{\sigma \sigma^{\prime}}$ the junction conductance for the $\sigma \sigma^{\prime}$ magnetic configuration and $P$ pointing in the $\alpha$ direction.

Firstly, it can be observed that our MTJ sustains a very robust TMR regardless of the direction of the polarization vector. This is simply a consequence of the spin-filtering effect and of the fact that the electronic structure of $\mathrm{SrTiO}_{3}$ and $\mathrm{BaTiO}_{3}$ are rather similar. More interesting is the dependence of the TER on the $\mathrm{SrTiO}_{3}$ barrier. Since in our junction the TER originates from a change in the $\mathrm{SrTiO}_{3}$ barrier height, the effect is expected to be magnified by increasing the barrier width, i.e., the $\mathrm{SrTiO}_{3}$ layer thickness. This is indeed the case, as demonstrated by the dependence of $R_{\text {TER }}$ on $m$ reported in Table I. In particular, we find that $R_{\text {TER }} \sim 0$ for $m=0$, i.e., when there is no intercalated $\mathrm{SrTiO}_{3}$. It then increases drastically for $m=1$ and $m=2$. This increase is, in fact, exponential in $m$, and it goes as $e^{-\left(\Delta^{\leftarrow}-\Delta^{\rightarrow}\right) m}$, where $\Delta^{\alpha}$ is the $\mathrm{SrTiO}_{3}$ barrier height in the $P_{\alpha}$ configuration. This is an important result, as it demonstrates that the TER can be tuned to a great degree by simply controlling the $\mathrm{SrTiO}_{3}$ layer thickness.

It is also important to note that for a given $(m \neq 0)$ junction there are four very distinct conductance states depending on both the magnetization direction of the electrodes and the polarization direction of the ferroelectric layer. This means that our proposed device can operate as a four state memory cell with four well-separated conductive states. Finally, one can quantify the dependence of the TMR on the $P$ direction by calculating the tunneling electro-magneto resistance (TEMR) ratio, defined as $R_{\mathrm{TEMR}}=\frac{R_{\mathrm{TMR}}^{\rightarrow}-R_{\mathrm{TMR}}^{\leftarrow}}{R_{\mathrm{TMR}}^{\leftarrow}}$. For $m=2$ we find $R_{\text {TEMR }}=460 \%$, a value which is comparable to those re- ported experimentally for $\mathrm{Fe} / \mathrm{BaTiO}_{3} / \mathrm{La}_{0.67} \mathrm{Sr}_{0.33} \mathrm{MnO}_{3}$ thin film structures (ranging between $140 \%$ and $450 \%$ ) [10].

In conclusion, we have discussed the effects of including a wide-band gap insulator into a MTJ based on a FE barrier. We have demonstrated that in such a junction the tunneling barrier profile can be tuned by reversing the direction of the macroscopic electrical polarization. This results in a tunable TER effect which may coexist with a TMR effect. In particular, the choice of $\mathrm{SrTiO}_{3}$ and $\mathrm{BaTiO}_{3}$, which both offer excellent spin-filtering to spins injected from $\mathrm{SrRuO}_{3}$, results in a device which also displays remarkably large TMR ratios. Importantly, both the TMR and the TER are tunable and increase with the $\mathrm{SrTiO}_{3}$ layer thickness. As such our proposed stack offers a robust protocol for constructing devices displaying simultaneous TER and TMR effects. These can operate as a four state memory element for data storage applications.

This work is sponsored by Science Foundation of Ireland (07/IN.1/I945) and by the EU-FP7 (ATHENA and iFOX projects). IR is sponsored by the King Abdullah University of Science and Technology (ACRAB project). Computational resources have been provided by the HEA IITAC project managed by TCHPC.

[1] S. Yuasa, T. Nagahama, A. Fukushima, Y. Suzuki and K. Ando, Nature Materials 3, 868 (2004)

[2] S.S.P. Parkin, C. Kaiser, A. Panchula, P.M. Rice, B. Hughes, M. Samant and S.-H. Yang, Nature Materials 3, 862 (2004).

[3] W.H. Butler, X.-G. Zhang, T.C. Schulthess and J.M. MacLaren, Phys. Rev. B 63, 054416 (2001).

[4] J. Mathon and A. Umerski, Phys. Rev. B 63, 220403(R) (2001).

[5] E.Y. Tsymbal and H. Kohlstedt, Science 313, 181 (2006).

[6] J.F. Scott, J. Mat. Chem., DOI:10.1039/ c2jm16137k, (2012).

[7] M. Gajek, M. Bibes, S. Fusil, K. Bouzehouane, J. Fontcuberta, A. Barthélémy and A. Fert, Nature Materials 6, 296 (2007).

[8] N.M. Caffrey, T. Archer, I. Rungger and S. Sanvito, Phys. Rev. B 83, 125409 (2011).

[9] J.P. Velev, C.-G. Duan, J.D. Burton, A. Smogunov, M.K. Niranjan, E. Tosatti, S.S. Jaswal and E.Y. Tsymbal, Nano Lett. 9, 427 (2009).

[10] V. Garcia, M. Bibes, L. Bocher, S. Valencia, F. Kronast, A. Crassous, X. Moya, S. Enouz-Vedrenne, A. Gloter, D. Imhoff, C. Deranlot, N.D. Mathur, S. Fusil, K. Bouzehouane and A. Barthélémy, Science 327, 1106 (2010).

[11] A. Crassous, V. Garcia, K. Bouzehouane, S.Fusil, A.H.G. Vlooswijk, G. Rispens, B. Noheda, M. Bibes and A. Barthélémy, Appl. Phys. Lett. 96, 042901 (2010).

[12] P. Maksymovych, S. Jesse, P. Yu, R. Ramesh, A.P. Baddorf and S.V. Kalinin, Science 324, 1421 (2009)

[13] J.M. Soler, E. Artacho, J.D. Gale, A. García, J. Junquera, P. Ordejón and D. Sánchez-Portal, J. Phys.: Con- 
dens. Matter 14, 2745 (2002).

[14] J.P. Perdew, K. Burke and M. Ernzerhof, Phys. Rev. Lett. 77, 3865 (1996).

[15] C.D. Pemmaraju, T. Archer, D. Sánchez-Portal and S. Sanvito, Phys. Rev. B 75, 045101 (2007).

[16] T. Archer, N.M. Caffrey and S. Sanvito, in preparation (2012).

[17] J.M. Rondinelli, N.M. Caffrey, S. Sanvito and N.A. Spaldin, Phys. Rev. B 78, 155107 (2008)

[18] M. Stengel, P. Aguado-Puente, N. A. Spaldin, and J. Junquera, Phys. Rev. B, 83235112 (2011).

[19] A. Filippetti, C.D. Pemmaraju, S. Sanvito, P. Delugas,
D. Puggioni and V. Fiorentini, Phys. Rev. B 84, 195127 (2011).

[20] A.R. Rocha, V.M. Garcia-Suárez, S.W. Bailey, C.J. Lambert, J. Ferrer and S. Sanvito, Phys. Rev. B. 73, 085414 (2006).

[21] A.R. Rocha, V.M. Garcia-Suárez, S.W. Bailey, C.J. Lambert, J. Ferrer and S. Sanvito, Nature Materials 4, 335 (2005).

[22] I. Rungger and S. Sanvito, Phys. Rev. B. 78, 035407 (2008).

[23] J. Junquera and P. Ghosez, Nature 422, 506 (2003) 